\title{
Desempenho agronômico e uso eficiente da terra em arranjos de plantas de mandioca e batata-doce
}

\author{
Amarílis Beraldo Rós ${ }^{1 *}$, Renata Espolador São João ${ }^{2}$ \\ 10.1590/0034-737X201663040012
}

\section{RESUMO}

O consórcio de duas ou mais culturas pode representar melhor uso do solo, alternativa para redução de perdas e incremento de renda. Assim, objetivou-se, com este trabalho, avaliar o desempenho agronômico de mandioca e batatadoce, consorciadas em diferentes arranjos, e determinar o índice de uso eficiente da terra (UET). O delineamento experimental foi em blocos ao acaso, com cinco tratamentos e cinco repetições. Os tratamentos foram: $\mathrm{M}$ (monocultivo de mandioca); 2M:1B (consórcio composto por duas leiras de mandioca alternadas com uma leira de batata-doce); 1M:1B (consórcio composto por leiras alternadas de mandioca e batata-doce); 1M:2B (consórcio composto por uma leira de mandioca alternada com duas leiras de batata-doce) e B (monocultivo de batata-doce). Cada parcela experimental foi constituída por seis ou oito fileiras de $7 \mathrm{~m}$ de comprimento, espaçadas de $0,9 \mathrm{~m}$ entre si, separadas por uma faixa de circulação de 2,0 m. Foram avaliados a produtividade comercial, a massa da matéria fresca individual, o comprimento, o diâmetro e a massa seca de raízes, sendo esses valores comparados pelo teste de Tukey $(\mathrm{p}<0,05)$. As maiores produtividades da mandioca ocorreram quando cultivada em M e 2M:1B, enquanto a maior produtividade de batata-doce foi em B. Os tratamentos estudados não apresentaram diferença significativa para massa da matéria fresca individual, comprimento, diâmetro e matéria seca de raiz de mandioca. Na batata-doce, o tratamento 1M:2B resultou em menor valor de massa da matéria fresca individual e de diâmetro de raiz. Constatou-se também vantagem do consórcio sobre o monocultivo, visto que todos os arranjos de consórcio promoveram valor de UET superior a 1. Logo, o consórcio entre mandioca e batata-doce resulta em melhor aproveitamento dos recursos ambientais, comparado com o monocultivo.

Palavras-chave: Ipomoea batatas (L.) Lam.; Manihot esculenta Crantz; UET; cultivo consorciado; morfologia de raiz.

\section{ABSTRACT}

\section{Agronomic performance and land equivalent ratio in arrangements of cassava and sweet potato plants}

Two or more cultures intercropped can have the best soil use, an alternative to reduce losses and increase income. This study aimed to evaluate the agronomic performance of cassava intercropped with sweet potato in different arrangements and determine the Land Equivalent Ratio (LER). The experimental design was randomized blocks with five treatments and five replications. The treatments were: $\mathrm{C}$ (cassava monoculture); $2 \mathrm{C}: 1 \mathrm{P}$ (two cassava rows intercropped with one sweet potato row); 1C:1P (one cassava row intercropped with one sweet potato row); 1C:2P (one cassava row intercropped with two sweet potato rows) and $\mathrm{P}$ (sweet potato monoculture). Each experimental plot consisted of 6 or 8 rows with $7 \mathrm{~m}$ length spaced $0.9 \mathrm{~m}$ from each other, and the experimental plots were separated by a $2.0 \mathrm{~m}$ space. The following were evaluated: commercial yield, individual fresh weight, length, diameter and dry mass of roots. The data were compared by Tukey test at 5\% probability. The cassava highest yields occurred in C and 2C:1P, while the sweet

\footnotetext{
Submetido em 23/04/2014 e aprovado em 29/12/2015.

Agência Paulista de Tecnologia dos Agronegócios, Presidente Prudente, São Paulo, Brasil. amarilis@apta.sp.gov.br

${ }^{2}$ Universidade do Oeste Paulista, Presidente Prudente, São Paulo, Brasil. res2tvp@ hotmail.com

* Autor para correspondência: amarilis@apta.sp.gov.br
} 
potato highest yield was in P. The treatments did not differ significantly for individual fresh mass, length, diameter and dry mass of cassava root. In sweet potato, the treatment $1 \mathrm{C}: 2 \mathrm{P}$ resulted in lower value of fresh mass and individual root diameter. There was also advantage of intercropping over monoculture because all arrangements with the intercropping promoted a LER value greater than 1. Thus, cassava intercropped with sweet potato results in better utilization of environmental resources compared to monoculture.

Key words: Ipomoea batatas (L.) Lam.; Manihot esculenta Crantz; LER; intercropping; root morphology.

\section{INTRODUÇÃO}

As culturas mandioca e batata-doce apresentam elevado potencial de produção, por apresentarem grande capacidade de produzir energia por unidade de área e de tempo, podendo produzir, segundo Rós et al. (2013) e Rós et al. (2012), cada uma, mais de $40 \mathrm{t} \mathrm{ha}^{-1}$, quando cultivadas em monocultivo e em condições favoráveis às culturas.

Essas culturas geralmente demandam espaçamento entre linhas de 0,8 a 1,0 m, mas, frequentemente, são cultivadas em monocultivo, principalmente a cultura da batata-doce. O cultivo em consórcio de mandioca com culturas de porte baixo ocorre com maior frequência, pois, durante parte de seu ciclo, a mandioca não apresenta alta percentagem de cobertura no solo (Otsubo \& Lorenzi, 2004) e, embora possam ser cultivadas em consórcios, há poucos estudos sobre o cultivo conjunto dessas duas culturas.

Como no consórcio há o cultivo simultâneo de duas ou mais culturas, deve-se dispor as plantas entre e dentro das fileiras de plantio, de modo a minimizar a competição, arranjando-as de forma que a distribuição espacial seja a mais favorável possível para todas as culturas. Schons et al. (2009) estudaram diferentes arranjos de plantas de mandioca e milho, em cultivos solteiro e consorciado, e verificaram expressivas diferenças de produtividade das culturas, em função da distribuição das plantas e de sua densidade, resultados semelhantes aos encontrados por Soares et al. (2011) com o consórcio de mandioca e feijão. Em consórcio batata-doce/quiabo, as culturas apresentaram produtividades semelhantes às verificadas em monocultivo, contudo houve alteração no número de raízes tuberosas de batata-doce produzidas (Njoku et al., 2007).

Dessa forma, para verificar se o consórcio está sendo mais produtivo que o cultivo solteiro, pode-se adotar o índice de uso eficiente da terra (UET). Njoku et al. (2007) concluíram, por meio do cálculo do uso eficiente da terra, que o consórcio batata-doce e quiabo resulta em maior produção que os monocultivos. No consórcio mandioca/ milho, em trabalho de Dapaah et al. (2003), as culturas tiveram sua produtividade reduzida em comparação ao monocultivo, no entanto o UET aumentou.

Assim, objetivou-se, com este trabalho, avaliar o desempenho agronômico dos consórcios entre mandioca e batata-doce, em diferentes arranjos, comparando-os aos cultivos solteiros das duas culturas.

\section{MATERIAL E MÉTODOS}

O experimento foi realizado na Agência Paulista de Tecnologia dos Agronegócios - Polo Alta Sorocabana, em Argissolo Vermelho-Amarelo, no município de Presidente Prudente, SP, a $22^{\circ} 11^{\prime}$ S e $51^{\circ} 23^{\prime} \mathrm{O}$, com 424,29 m de altitude. O clima regional é classificado como Aw, apresentando duas estações bem definidas: verão quente e úmido e inverno ameno e seco. Os dados meteorológicos obtidos durante a condução do experimento encontramse na Figura 1.

As análises química e física da camada de 0-20 m, realizadas em amostra do solo obtida antes da instalação do experimento resultaram em: $\mathrm{pH}\left(\mathrm{CaCl}_{2}\right)$ de 5,$1 ; 32 \mathrm{mg} \mathrm{dm}^{-3}$ de P; 3,3 mmol $\mathrm{dm}^{-3}$ de $\mathrm{K}^{+} ; 0,0 \mathrm{mmol}_{\mathrm{c}} \mathrm{dm}^{-3} \mathrm{de}^{+3} ; 18$

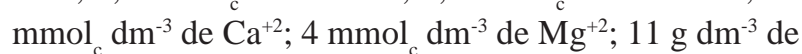
matéria orgânica; $25 \mathrm{mmol} \mathrm{dm}_{\mathrm{c}}^{-3} \mathrm{de}$ Soma de Base; $44 \mathrm{mmol}_{\mathrm{c}}$ $\mathrm{dm}^{-3}$ de CTC e 57\% de Saturação de Bases; $900 \mathrm{~g} \mathrm{~kg}^{-1} \mathrm{de}$ areia; $40 \mathrm{~g} \mathrm{~kg}^{-1}$ de silte e $60 \mathrm{~g} \mathrm{~kg}^{-1}$ de argila. Não foi realizada adubação do solo.

A área de plantio foi preparada por meio de aração e gradagem niveladora. Com o intuito de facilitar o desenvolvimento radicular das culturas e viabilizar o cultivo da batata-doce, foram confeccionadas leiras com cerca de $0,35 \mathrm{~m}$ de altura, com sulcador. As leiras foram utilizadas pelas duas culturas.

As culturas foram plantadas, simultaneamente, em 29 de setembro de 2012. Foram utilizados o cultivar de mandioca IAC 576-70, que apresenta médio porte e ramificação alta e aberta (Irolivea et al., 1998), e o cultivar de batata-doce Uruguaiana, ambos amplamente utilizados na região. Para o plantio da mandioca, foram retiradas manivas com 0,2 m de comprimento, obtidas do terço médio de hastes de plantas com dez meses de idade. As manivas foram plantadas a $0,1 \mathrm{~m}$ de profundidade, em leiras. Para o 
plantio da batata-doce, foram utilizados segmentos de ramas com cerca de $0,3 \mathrm{~m}$ de comprimento, oriundas de ponteiros de plantas de batata-doce originadas de material vegetativo de plantas matrizes isentas de vírus. Os segmentos de ramas foram inseridos verticalmente nas leiras por meio de abertura manual de orifícios com 0,08 $\mathrm{m}$ de profundidade, sendo parcialmente enterradas manualmente.

O delineamento experimental foi em blocos casualizados, com cinco tratamentos e cinco repetições. Os tratamentos foram compostos por cinco arranjos das culturas de mandioca e batata-doce, em cultivos solteiros e consorciados. Os tratamentos foram: monocultivo de mandioca (M), com 15.873 plantas ha ${ }^{-1}$; cultivo consorciado, composto por duas leiras de mandioca, alternadas com uma leira de batata-doce (2M:1B), com 10.582 plantas ha${ }^{1}$ de mandioca e 10.582 plantas ha ${ }^{-1}$ de batata-doce; cultivo consorciado, composto por leiras alternadas de mandioca e de batata-doce (1M:1B) com 7.936 plantas ha $^{-1}$ de mandioca e 15.873 plantas ha ${ }^{-1}$ de batata-doce; cultivo consorciado, composto por uma leira de mandioca alternada com duas leiras de batata-doce (1M:2B), com 5.291 plantas ha-1 de mandioca e 21.164 plantas $^{-1} \mathrm{a}^{-1}$ de batatadoce; monocultivo de batata-doce (B), com 31.746 plantas $\mathrm{ha}^{-1}$. Dessa forma, foram implantados consórcios substitutivos, baseados em porcentagens da população do monocultivo de cada cultura, sendo $\mathrm{M}$ equivalente a 100\%M; 2M:1B (66\%M:33\%B); 1M:1B, (50\%M:50\%B); 1M:2B (33\%M:66\%B) e B, 100\%B.

Cada parcela experimental foi constituída por seis ou oito fileiras de $7 \mathrm{~m}$ de comprimento, espaçadas de $0,9 \mathrm{~m}$ entre si, separadas por uma faixa de circulação de $2,0 \mathrm{~m}$. O número de plantas por fileira dependeu da cultura: dez plantas de mandioca ( $0,7 \mathrm{~m}$ entre plantas) e 20 plantas de batata-doce ( $0,35 \mathrm{~m}$ entre plantas).

A área útil das parcelas foi constituída por, no mínimo, duas fileiras centrais de cada cultura, excetuando-se as plantas das extremidades. A área experimental foi mantida sem a presença de plantas infestantes por meio de capinas manuais durante todo o ciclo das culturas.

Durante a condução do experimento, a cada 14 dias, as alturas médias das plantas de mandioca, durante o período de 30 a 100 dias após plantio (DAP), pela tomada de medida da porção compreendida entre o nível do solo e o limbo da folha mais alta.

A colheita da batata-doce ocorreu aos 130 DAP e foi avaliada a produtividade comercial de raízes tuberosas (massa da matéria fresca entre 80 e $1000 \mathrm{~g}$ e formato uniforme e liso). Também foram avaliados a massa da matéria fresca individual, o comprimento e o diâmetro das raízes tuberosas consideradas comerciais e a massa da matéria seca de 20 raízes, coletadas ao acaso, em cada parcela. A massa da matéria seca foi obtida por meio da secagem da porção mediana picada das raízes, em estufa a $60^{\circ} \mathrm{C}$, até massa constante.

A mandioca foi colhida aos 270 DAP, sendo consideradas todas as raízes saudáveis, com comprimento e diâmetro iguais ou superiores a 10 e a $3 \mathrm{~cm}$, respectivamente. Foram selecionadas ao acaso 20 raízes de mandioca, por parcela, para avaliação da massa da matéria fresca individual, do comprimento, do diâmetro das raízes e da massa da matéria seca. Também foi determinada a massa da matéria fresca da parte aérea das plantas de mandioca, por meio da pesagem de cinco plantas por parcela.

Para obtenção do índice do uso eficiente da terra (UET), foi realizado o cálculo: $\mathrm{UET}=(\mathrm{RMaC} / \mathrm{RMaS})+$ ( $\mathrm{RBaC} / \mathrm{RBaS}$ ), em que RMaC é a produtividade de raízes da mandioca em consórcio; RMaS, a produtividade de raízes da mandioca cultivada de forma solteira (M); $\mathrm{RBaC}$, a produtividade de raízes tuberosas de batata-doce em consórcio; $\mathrm{RBaS}$, a produtividade de raízes tuberosas de batata-doce cultivada na forma solteira (B).

Os dados referentes à altura das plantas de mandioca foram submetidos a análises de variância (ANOVA) em

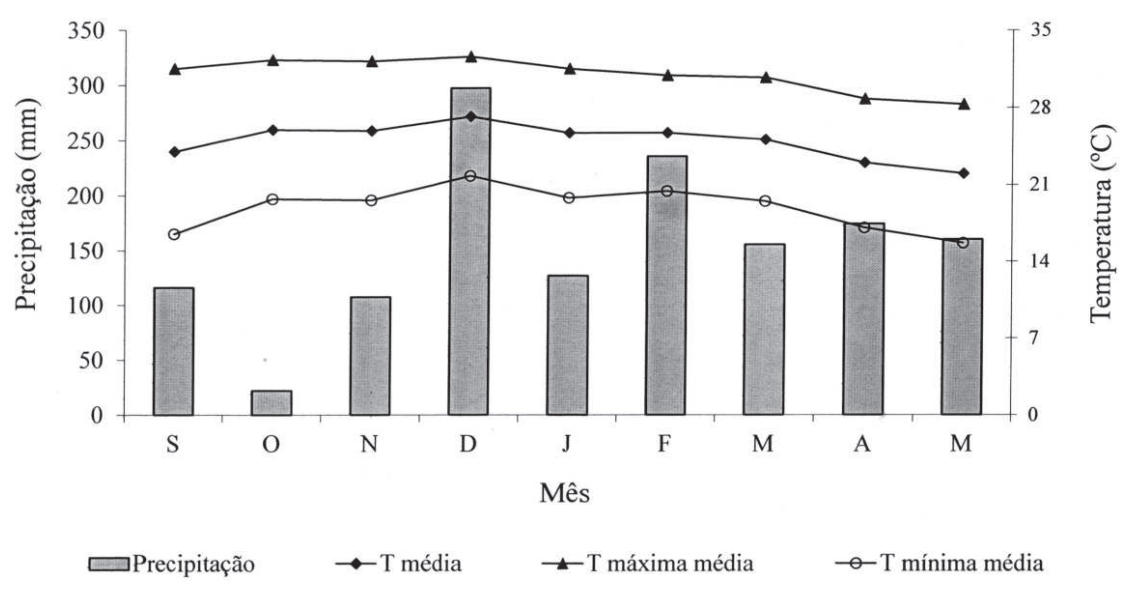

Figura 1: Precipitação, temperaturas média, máxima média e mínima média, ocorridas de setembro de 2012 a maio de 2013. 
esquema de parcela subdividida no tempo. Posteriormente, foi aplicada a análise de regressão em função dos dias após o plantio, utilizando-se os valores médios. As médias das demais variáveis foram comparadas pelo teste de Tukey $(\mathrm{p}<0,05)$. Os dados referentes a UET são descritivos e não foram analisados estatisticamente.

\section{RESULTADOS E DISCUSSÃO}

Segundo a análise estatística, não houve interação significativa entre os arranjos de plantas e as épocas de avaliação, para a característica altura de plantas de mandioca. Os arranjos não resultaram em diferenças estatísticas, ou seja, os consórcios de mandioca com batata-doce não influenciaram a altura das plantas de mandioca, que apresentou valor médio de 0,64 m. Schons et al. (2009), de maneira semelhante, verificaram que a altura de plantas de mandioca não foi afetada pela presença de plantas de milho em plantio consorciado.

A altura de plantas de mandioca foi influenciada pelos DAP, seguindo modelo linear crescente. Observa-se que aos 30 DAP, as plantas de mandioca apresentaram 0,15 m e aos 100 DAP passaram para 1,14 m (Figura 2). Esse resultado era esperado, pois, no período em que foram tomadas as medidas, as condições de temperatura e precipitação estavam favoráveis ao crescimento das plantas.

Quanto à produtividade, houve diferença significativa entre os tratamentos em ambas as culturas (Tabela 1). As maiores produtividades de raízes de mandioca ocorreram em M e em 2M:1B. Assim, a redução em até 34\% na população de mandioca quando em consórcio com batata-doce não resulta em queda de produtividade, diferentemente do que ocorre com reduções de $50 \%$ e $66 \%$ na população de plantas. A produção por unidade de planta também foi afetada (Tabela 1). Nos tratamentos em que houve o consórcio (2M:1B, 1M:1B e 1M:2B), as plantas de mandioca produziram mais individualmente que as plantas em monocultivo, ou seja, as plantas cultivadas em espaçamento entre linhas igual ou superior a 1,8 m acu-

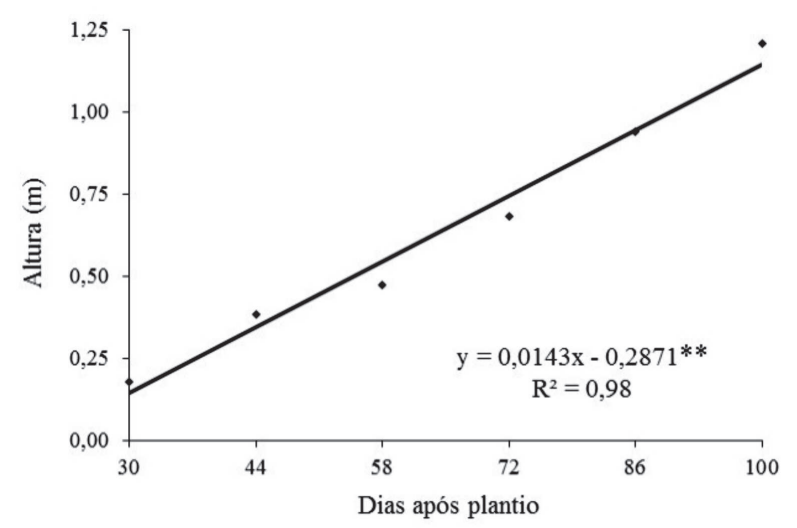

Figura 2: Altura de plantas de mandioca em função de dias após plantio. ${ }^{* *}$ Significativo a $1 \%$ pelo teste $\mathrm{F}$. mularam maior quantidade de substâncias reserva em seu sistema radicular. Tal fato pode estar associado à maior competição entre plantas de mandioca em monocultivo, bem como a um aumento no número de raízes por planta, visto que Guerra et al. (2005), estudando duas cultivares de mandioca, obtiveram maior produtividade de raízes no maior espaçamento, em função do maior número de raízes tuberosas por unidade de planta.

Segundo Barros et al. (1978), a produção por planta da mandioca é controlada pela capacidade de dreno das raízes, a qual é maior em menores densidades de plantio, em que há mais espaço disponível para as raízes. Aguiar et al. (2011) também verificaram que plantas individuais submetidas à menor competição têm maior produção de raízes.

Em trabalho de Devide et al. (2009), não houve diferença de produtividade de mandioca em monocultivo e consorciada com caupi e milho, o que está associado a manutenção do mesmo estande de mandioca em todos os tratamentos, diferentemente do observado neste trabalho no qual o estande diferiu entre os tratamentos.

A produtividade da batata-doce também variou em função dos tratamentos (Tabela 1). A cultura apresentou maior diferença na produtividade nos diferentes arranjos, que a cultura da mandioca. Houve maior produtividade de raízes em monocultivo (B), com redução significativa de produtividade a cada diminuição da densidade das plantas de batata-doce. Bezerra et al. (2007) também quantificaram diferenças de produtividade nos consórcios sorgo e feijão-de-corda e sorgo e milho, em função das proporções de cada espécie. No entanto, a produtividade de raízes pode ser favorecida por cultivo consorciado, conforme verificado em trabalhos de Cecílio Filho \& May (2002) e Rezende et al. (2003), nos quais rabanetes, cultivados com alface, apresentaram maior produção de raízes comerciais em sistema consorciado, o que foi relacionado com o efeito favorável da alface na cobertura do solo.

Ressalta-se que a elevada produtividade de raízes tuberosas de batata-doce, obtida neste trabalho, tanto em monocultivo quanto em consórcio, deve-se, provavelmente, à elevada sanidade do material de plantio (oriundo de matrizes isentas de vírus) e a condições edafoclimáticas bastante adequadas à cultura.

A produção de raízes de batata-doce por unidade de planta (Tabela 1) foi maior em monocultivo, diferentemente do ocorrido com a cultura da mandioca. A presença de plantas de mandioca resultou em menor produção por planta, independentemente do arranjo entre plantas. Essa diminuição de produtividade provavelmente é justificada pelo maior sombreamento causado pelas plantas de mandioca, que apresentam porte mais elevado e hastes ramificadas. A cultura da mandioquinha-salsa, em avaliação de Tolentino Junior et al. (2002), apresentou maior número de raízes comerciais por unidade de planta em 
Tabela 1: Produtividade de mandioca e batata-doce em monocultivo e consorciadas

\begin{tabular}{|c|c|c|c|c|}
\hline \multirow{2}{*}{ Tratamento } & \multicolumn{2}{|c|}{ Produtividade $^{*}\left(\mathrm{t} \mathrm{ha}^{-1}\right)$} & \multicolumn{2}{|c|}{ Produtividade $^{*}\left(k_{\text {k planta }}{ }^{-1}\right)$} \\
\hline & Mandioca & Batata-doce & Mandioca & Batata-doce \\
\hline $\bar{M}$ & $32,2 \mathrm{~A}$ & - & $2,03 \mathrm{~B}$ & - \\
\hline $2 \mathrm{M}: 1 \mathrm{~B}$ & $28,4 \mathrm{~A}$ & $11,1 \mathrm{D}$ & $2,68 \mathrm{~A}$ & $1,05 \mathrm{~B}$ \\
\hline $1 \mathrm{M}: 1 \mathrm{~B}$ & $21,6 \mathrm{~B}$ & $17,4 \mathrm{C}$ & $2,65 \mathrm{~A}$ & $1,09 \mathrm{~B}$ \\
\hline $1 \mathrm{M}: 2 \mathrm{~B}$ & $16,3 \mathrm{C}$ & $22,4 \mathrm{~B}$ & $3,09 \mathrm{~A}$ & $1,06 \mathrm{~B}$ \\
\hline $\mathrm{B}$ & - & $41,3 \mathrm{~A}$ & - & $1,30 \mathrm{~A}$ \\
\hline $\mathrm{CV}(\%)$ & 11,30 & 5,44 & 14,23 & 8,67 \\
\hline
\end{tabular}

*Letras diferentes na coluna diferem entre si pelo Teste de Tukey a 5\% de probabilidade de erro.

cultivo solteiro, em comparação com os consórcios com beterraba e alface, o que resultou maior produtividade no monocultivo, enquanto, em trabalho de Njoku et al. (2007), a produtividade de raízes tuberosas de batata-doce em monocultivo e consorciada com quiabeiro não variou.

Analisando-se as demais variáveis, não se observam diferenças estatísticas para as características massa da matéria fresca individual, comprimento, diâmetro e matéria seca de raiz de mandioca, sendo os valores médios: $0,561 \mathrm{~kg} ; 34,4 \mathrm{~cm} ; 5,2 \mathrm{~cm}$ e $42,3 \%$, respectivamente. Em estudo de Albuquerque et al. (2012), mandioca em monocultivo e consorciada com feijão comum em fileira simples, em iguais arranjos espaciais de plantas de mandioca, também apresentaram semelhantes valores de comprimento de raiz e de matéria seca de raiz; contudo, o diâmetro de raiz foi favorecido no cultivo consorciado.

Para batata-doce, também, não houve diferenças para as características comprimento e matéria seca de raiz, sendo os valores médios: $20,4 \mathrm{~cm}$ e $26,1 \%$, respectivamente, mas houve diferença entre os tratamentos nas características massa da matéria fresca individual e diâmetro de raiz (Tabela 2). De maneira semelhante, Tolentino Junior et al. (2002) observaram redução do diâmetro de raiz de beterraba em cultivo consorciado com mandioquinha-salsa, em comparação com as mesmas características no seu monocultivo, o que foi justificado pela maior competição e menor aproveitamento do solo pelas plantas, quando em cultivo consorciado.

Tabela 2: Diâmetro médio e massa da matéria fresca individual de raízes de batata-doce

\begin{tabular}{lcc}
\hline Tratamento & $\begin{array}{c}\text { Massa da matéria } \\
\text { fresca individual } \\
\text { de raiz }(\mathbf{g}) *\end{array}$ & $\begin{array}{c}\text { Diâmetro de raiz } \\
(\mathbf{c m}) *\end{array}$ \\
\hline $1 \mathrm{M}: 2 \mathrm{~B}$ & $422 \mathrm{AB}$ & $6,9 \mathrm{~A}$ \\
$\mathrm{~B}$ & $475 \mathrm{~A}$ & $6,7 \mathrm{~A}$ \\
$1 \mathrm{M}: 1 \mathrm{~B}$ & $369 \mathrm{AB}$ & $6,2 \mathrm{AB}$ \\
$2 \mathrm{M}: 1 \mathrm{~B}$ & $292 \mathrm{~B}$ & $5,5 \mathrm{~B}$ \\
\hline $\mathrm{CV}(\%)$ & 10,48 & 23,03 \\
\hline
\end{tabular}

*Letras diferentes na coluna diferem entre si pelo Teste de Tukey a $5 \%$ de probabilidade de erro.
Em 2M:1B, as raízes da batata-doce apresentaram menor diâmetro e, consequentemente, menor massa da matéria fresca individual de raiz que em $\mathrm{B}$, enquanto, nos demais arranjos de consórcio, os valores foram semelhantes aos do monocultivo de batata-doce. Esse resultado pode estar relacionado com o maior sombreamento da cultura, quando há mandioca cultivada em fileiras duplas, em comparação com os demais arranjos entre as culturas.

Quanto à massa da matéria fresca da parte aérea das plantas de mandioca, não houve diferenças entre os tratamentos (média de 2,77 kg planta ${ }^{-1}$ ). Devide (2006), estudando mandioca em monocultivo e consorciada com milho e caupi, também verificou que não houve diferenças na produção da massa da parte aérea entre os tratamentos.

$\mathrm{Na}$ avaliação do uso eficiente da terra, constatou-se vantagem do consórcio sobre o monocultivo (Tabela 3), visto que todos os arranjos de consórcio promoveram valor de UET superior a 1 .

Em 2M:1B, há necessidade de uma área $15 \%$ maior para obter-se a mesma produtividade de mandioca e batata-doce em monocultivo. No consórcio mandioca e feijãocaupi, os dados de UET, obtidos por Albuquerque et al. (2012), revelam que são necessários de 1,28 ha a 1,54 ha em cultivo das culturas de mandioca e feijão-caupi de forma solteira para obter as produtividades equivalentes a 1,0 ha de cultivo consorciado. Ressalta-se que o valor de UET, obtido no trabalho de Albuquerque et al. (2012), foi superior ao verificado neste trabalho, mas naquele não houve redução da densidade plantas de mandioca quando em consórcio, diferentemente do que ocorreu neste trabalho.

Tabela 3: Uso Eficiente da Terra (UET) nos diferentes arranjos de consórcio

\begin{tabular}{lccc}
\hline \multirow{2}{*}{ Tratamento } & \multicolumn{2}{c}{ UET parcial } & \multirow{2}{*}{ UET } \\
\cline { 2 - 3 } & Mandioca & Batata-doce & \\
\hline 2M:1B & 0,88 & 0,27 & 1,15 \\
1M:1B & 0,67 & 0,42 & 1,09 \\
1M:2B & 0,51 & 0,54 & 1,05 \\
\hline
\end{tabular}

Rev. Ceres, Viçosa, v. 63, n.4, p. 517-522, jul/ago, 2016 
Em consórcio entre mandioca e milho, Devide et al. (2009) verificaram que a produtividade da cultura da mandioca consorciada foi semelhante à da mandioca em monocultivo e que a produção de milho representou renda adicional ao produtor. No entanto, consórcios de mandioca com Stizolobium deeringeanum e com Crotalaria grantiana promoveram queda de produtividade de mandioca, ainda que tenha sido mantida a mesma densidade de plantas de mandioca encontrada no monocultivo (Takahashi \& Bicudo, 2009).

Dessa forma, o consórcio com diferentes arranjos entre espécies pode promover alterações na produtividade das culturas envolvidas, resultando em maior produtividade por unidade de área. No entanto, deve-se associar a produtividade de cada cultura ao seu possível valor comercial no momento da colheita, de forma a avaliar se o melhor uso eficiente da terra resulta em ampliação de renda.

\section{CONCLUSÕES}

Há decréscimo de produtividade das culturas consorciadas na maioria dos arranjos, quando comparada com as de seus respectivos monocultivos.

Os arranjos de consórcio estudados são mais produtivos que os cultivos solteiros.

A produção por planta de mandioca em monocultivo é menor que em consórcio devido à menor densidade populacional verificada nos consórcios, o que resulta em menor competição entre plantas.

A produção por planta de batata-doce é maior quando adotado o monocultivo.

\section{AGRADECIMENTO}

As autoras agradecem ao Conselho Nacional de Desenvolvimento Científico e Tecnológico (CNPq), pela bolsa do Programa Institucional de Bolsas de Iniciação em Desenvolvimento Tecnológico e Inovação (PIBITI), concedida à segunda autora.

\section{REFERÊNCIAS}

Aguiar EB, Valle TL, Lorenzi JO, Kanthack RAD, Miranda Filho H \& Granja NP (2011) Efeito da densidade populacional e época de colheita na produção de raízes de mandioca de mesa. Bragantia, 70:561-569.

Albuquerque JAA, Sediyama T, Alves JMA, Silva AA \& Uchôa SCP (2012) Cultivo de mandioca e feijão em sistemas consorciados realizado em Coimbra, Minas Gerais, Brasil. Revista Ciência Agronômica, 43:532-538.

Barros RS, Mercês WC \& Alvim R (1978) Sink strength and cassava productivity. HortScience, 13:474-475.

Bezerra APA, Pitombeira JB, Távora JA \& Vidal FC (2007) Rendimento, componentes da produção e uso eficiente da terra nos consórcios sorgo x feijão-de-corda e sorgo x milho. Revista Ciência Agronômica, 38:104-108.
Cecílio Filho AB \& May A (2002) Produtividade das culturas de alface e rabanete em função da época de estabelecimento do consorcio, em relação aos monocultivos. Horticultura Brasileira, 20:501-504.

Dapaah HK, Asafu-Agyei JN, Ennin AS \& Yamoah C (2003) Yield stability of cassava, maize, soya bean and cowpea intercrops. The Journal of Agricultural Science, 140:73-82.

Devide ACP, Ribeiro RLD, Valle TL, Almeida DLA, Castro CM \& Feltran JC (2009) Produtividade de raízes de mandioca consorciada com milho e caupi em sistema orgânico. Bragantia, 68:145153.

Devide ACP (2006) Sistema orgânico de produção de mandioca consorciada com milho e caupi. Dissertação de Mestrado. Universidade Federal Rural do Rio de Janeiro, Seropédica. 85p.

Guerra AF, Fialho JJ \& Rocha OC (2005) Produtividade e qualidade de raízes de mandioca em resposta ao regime hídrico e a densidades de plantio. In: XI Congresso Brasileiro de Mandioca, Campo Grande. Anais, Sociedade Brasileira de Mandioca. CDRom.

Irolivea EAM, Camara GMS, Nogueira MCS \& Cintra HS (1998) Efeito do espaçamento entre plantas e da arquitetura varietal no comportamento vegetativo e produtivo da mandioca. Scientia Agricola, 55:269-275.

Njoku SC, Muoneke CO, Okpara DA \& Agbo FMO (2007) Effect of intercropping varieties of sweet potato and okra in an ultisol of southeastern Nigeria. African Journal of Biotechnology, 6:1650-1654.

Otsubo AA \& Lorenzi JO (2004) Cultivo da mandioca na região Centro-Sul do Brasil. Dourados, Embrapa Agropecuária Oeste. $51 \mathrm{p}$.

Rezende BLA, Canato GHD \& Cecílio Filho AB (2003) Productivity of lettuce and radish cultivations as a function of spacing and of time of establishment of intercropping. Acta Horticulturae, 607:97-101.

Rós AB, Hirata ACS \& Narita N (2013) Produção de raízes de mandioca e propriedades química e física do solo em função de adubação com esterco de galinha. Pesquisa Agropecuária Tropical, 43:247-254.

Rós AB, Hirata ACS \& Santos HS (2012) Avaliação da produtividade de plantas de batata-doce oriundas de matrizes livres de vírus. Agrária, 7:434-439.

Schons A, Streck NA, Storck L, Buriol GA, Zanon AJ, Pinheiro DG \& Kraulich B (2009) Arranjos de plantas de mandioca e milho em cultivo solteiro e consorciado: crescimento, desenvolvimento e produtividade. Bragantia, 68:155-167.

Soares MBS, Finotto EL \& Martins ALM (2011) Produtividade e eficiência do uso da terra no consórcio entre mandioca e amendoim. Disponível em: <http://www.aptaregional.sp.gov.br/ acesse-os-artigos-pesquisa-e-tecnologia/edicao-2011/2011-julho-dezembro/1121-produtividade-e-eficiencia-do-uso-da-terrano-consorcio-entre-mandioca-e-amendoim/file.html>. Acessado em: 14 de março de 2016.

Takahashi M \& Bicudo SJ (2009) Consorciação da mandioca em dois arranjos de plantas com duas espécies leguminosas. In: XIII Congresso brasileiro de Mandioca, Botucatu. Anais, Sociedade Brasileira da Mandioca. CD-Rom.

Tolentino Junior CF, Zárate NA \& Vieira MC (2002) Produção da mandioquinha-salsa consorciada com alface e Beterraba. Acta Scientiarum, 24:1447-1454.

Willey RW (1979) Intercropping: its importance and research needs. Part 1. Competition and yield advantages. Field Crops Abstract, 32:1-10. 